\title{
REPORT OF WORKING GROUP ON
}

\section{HARMONIZATION AND IMPROVING}

\author{
DRUG SUPPLY \& USE
}

\section{BY THE AID COMMUNITY IN AFGHANISTAN}




\title{
REPORT OF WORKING GROUP ON
}

\author{
HARMONIZATION AND IMPROVING
}

\author{
DRUG SUPPLY \& USE
}

BY THE AID COMMUNITY IN AFGHANISTAN 


\section{MEMBERS OF WORKING GROUP:}

Dr. Almud F Danish. Temporary Consultant UNICEF (Rapportcur)

Mr. Assadullah. Manager Aıcein Pharmaccutical lnstitute. Kabul

Dr Azizullah Akhgar. President. Kabul Medical Institute

Ms.E. Spencer. Progranmm Officer Agency Coordinating Bods for Afghan Relief (ACBAR)

(Moderator)

Dr Essmatullah. Programme Manager. Nonwegian Afghanistan Committec (NAC) (Rapportcur)

Dr Jama.A.M * Medical Officer. WHO

Dr. Loan Licm .* Technical Coordinator. Ibn Sina

Dr.Mirza Jan. Director. Hcalth, Islamic Relief Agency (ISRA)

Mr. Mohammad. Hakimi. Head of Inspection \& Regulation. Ministry of Public Health, Kabul

Dr. Mohammad Saced, Technical Adviser.Health. Swedish Committec for Afghanistan (SCA)

Dr.Mohammad.Shoaib.Abedi. Asst.Technical coordinator. Ibn Sina

Dr.Mahammad Shoaib.Ahmadzai. Training Adviser. Aide Medicale Internationale (AMI)

Dr.Padsha. Salch.* Bednet Project Manager. Medicins San Frontiers (MSF)

*: only attended some scssions.

\section{ACKNOWLEDGMENT:}

ACBAR Health Sub-committee and the working grouj) would like to thank UNICEF/ Afghanistan Programme Office for funding this Workshop. 


\section{TABLE OF CONTENTS}

I.SUMMARY

2 INTRODUCTION

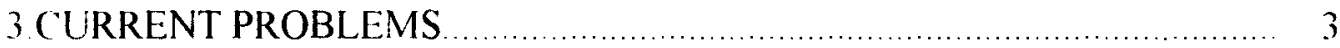

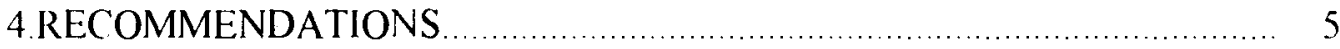

- SELECTION \& QUANTIFICATION .......................................... 5

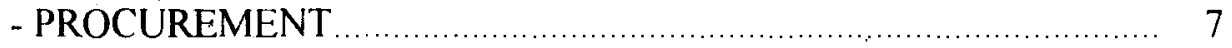

- QUALITY ASSURANCE _........................................................ 9

- STORAGE \& DISTRIBUTION ….............................................. 9

- USE

- APPENDIX I FURTHER READING ............................................... 11

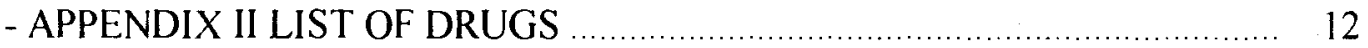




\section{SUMMARY:}

Afghanistan is faced with serious health problems. Health needs exceed the financial resources. Health services and drugs are provided by the public sector, the private sector and by the aid community in Afghanistan. About 54 national and international organisations are working in the health sector in Afghanistan.

At present drugs are procured by the aid community in a decentralised form and each organisation selects the drugs of their choice. While some organisations provide effective, safe and good quality drugs at reasonable prices, others face difficulties in this domain. In some circumstances drug donations create problems rather than benefits. Few organisations have access to quality control tests or obtain valid certifications on the quality of drugs.

Irrational drug prescribing that can nullify all the efforts. This is a crucial problem in all facilities including hospitals and sub-centres.

In order to harmonize and rationalise the drug supply by the aid community and to promote rational drugs use, a working group was established through ACBAR Health Sub-Committee with financial and technical cooperation of UNICEF. The working group organised a five day Workshop, December 8-12, 1996.

The working group, evaluated the current situation of drug supply and use within the aid community in detail; and based on the National List of Essential Drugs, prepared uniform drugs lists for different health facilities for use by the aid community.

The working group identified a number of unsafe/unnecessary drugs and recommended those not to be supplied. In order to improve the supply system, a centralised procurement system or joint purchasing was recommended. In addition a number of recommendations for improving drug donation, quality assurance, storage, distribution, prescribing, dispensing and drug use by patients were made. 


\section{INTRODUCTION:}

\subsection{BACKGROUND:}

Afghanistan being one of the least developed countries in the world and faced with long civil unrest, is also faced with serious health problems. Health needs exceed its financial resources. Health services are provided by the public sector, the private sector and the aid community.

About 54 national and international organisations are working in the health sector in Afghanistan. (1) It is estimated that some US\$12 Million are spent in this area. Drugs are also provided through these organisations. Some organisations are only involved in drug donation while others besides drug donation, run health facilities where drugs are prescribed and dispensed. The financial capacity also differs greatly among organisations.

At present drugs are procured by the aid community in a decentralised form, i.e., every organisation selects their own drugs. In those organisations running health facilities, the process of drug supply including selection, quantification, procurement, storage, distribution, transportation, prescribing and dispensing take place within that organisation.

While some organisations provide effective, safe and good quality drugs at reasonable prices, others face difficulties in this domain. A number of unnecessary and unsafe drugs with irrational formulations are procured by some organisations. In certain circumstances drug donations can produce problems rather than benefits in. Drugs use including prescribing, dispensing and use by patients is not satisfactory, in many instances, which can in turn produce more problems.

The public central drug supply department and the central quality control laboratory in Kabul have been destroyed reducing the possibility of quality control within Afghanistan.

Due to the above reasons the aid community involved in the health sector requested ACBAR through ACBAR Health Sub-Committee, to establish a working group on the harmonisation and rationalisation of drug supply. ACBAR with financial and technical cooperation of UNICEF organised a working group from December 8-12, 1996.

(1) ACBAR Data base, Agency Coordinating Body Afghan Relief 1995 


\subsection{OBJECTIVES:}

The objectives of the working group were:

- To maximize the use of available resources in procurement of safe, effective, useful and good quality drugs on a regular basis by the aid community.

Promoting rational drugs use

Protect the health of people against the hazards of unsafe and ineffective drugs and from irrational use of drugs.

\section{CURRENT PROBLEMS:}

\subsection{SELECTION \& QUANTIFICATION:}

The majority of organisations do not have the required staff for satisfactory selection and quantification. A number of unnecessary drugs, like combined antidiarrhoeal paediatric formulations, multivitamins, irrational fixed ratio combinations and other drugs that are banned or withdrawn in other countries like dipirone and phenylbutazone are provided by some organisations. Inaccurate quantification results, either in under-prescribing or accumulation and expiration of drugs.

\subsection{PROCUREMENT \& QUALITY ASSURANCE:}

Drugs are procured in a decentralized manner through tenders, direct purchase or donation. Few organisations have access to quality control tests or valid certification on the quality of drugs. Organisations with limited financial capacity who procure drugs through direct purchase from whole-salers or intermediate dealers are faced with crucial problems. 


\subsection{STORAGE \& DISTRIBUTION}

The majority of organisations lack suitable warehouse storage facilities at central and peripheral levels. Good and safe systems of stock keeping and distribution are practised in all organisations. Sometimes administerial problems regarding custom clearance, transportation and other documentation are experienced.

\subsection{USE OF DRUGS}

Irrational drug prescribing can nullify all efforts and is a crucial problem in all facilities including hospitals and other health facilities. Over-prescribing of antibiotics, analgesics; misuse of injections and psychotherapeutic; and multiple prescribing as well as under-prescribing are widespread. The majority of prescribers do not have access to unbiased and reliable drugs information references.

Drug dispense at health facilities is often disrupted by patient pressure i.e some patients urge dispensers to give them their desired drugs or dosage forms other than that prescribed.

Although no study has been done in the field of drug use by patients, reports say that patients drug information is very low and their perception on drugs relies more; on a pill for every ill conception.

\section{RECOMMENDATIONS}

\subsection{SELECTION \& QUANTIFICATION:}

The working group used the National List of Essential Drugs [2] as the basis for the drugs list to be recommeded for use by aid community. Taking into consideration financial resources priority was given to the availability of pharmaceutical products of proven efficacy, safety and those that satisfy the real health needs of the population. The choice of drugs was based on many factors: such as the pattern of prevalent diseases; the treatment facilities; the training and experiences of available personnel; financial resources and genetic, demographic and environmental factors. Separate limited lists were prepared for National/Regional hospitals, Provincia/District hospitals, Basic Health Centre/C1 Sub-centre/C2 (see Appendix I page 11). 
Due to the vast differences between health facilities, organisations' policies and financial resources, the issue of quantification was left to be determined by the organisation themselves. The working group recommends that the quantification should be based on the number of population covered by the health facility, morbidity rate, the standard drug treatment schedules and the ratio of adults/children

The following recommendations are made by the working group, regarding drug selection:

\subsection{1.}

Due to the severe side-effects and or ineffectiveness, the following drugs should not be procured:

- $\quad$ Antidiarrhoeal paediatric formulations including Kaolin, Pectin, Loperamide, Diphenoxylate

- Dipironel Analgin,

- Paediatric Aspirin

- Phenylbutazone and Oxyphenbutazone

- Multivitamins e.i., Two or more than two vitamins with or without other ingredients

\subsubsection{Fixed-ratio combinations:}

Only a limited number of fixed-ratio combinations are acceptable. Many fixed-ratio combinations are irrational and may cause several problems. According to WHO [3], Fixed-ratio combination products are acceptable only when the dosage of each ingredient meets the requirement of a defined population group and when the combination has a proven advantage over single compounds administered separately in therapeutic effect, safety or compliance. 


\subsubsection{Drug donation:}

Drug donations should be in line with WHO Guidelines for drug donation [4]. The main points from this guideline are as follows:

- $\quad$ All drug donations should be based on an expressed need and be relevant to the diseases pattern in the recipient country.

- $\quad$ All donated drugs should be approved for use in the recipient country and appear on the national list of essential drugs or if a national list is not available, on the WHO model list of essential drugs.

- $\quad$ The presentation, strength and formulation of donated drugs should, as far as possible, be similar to those commonly used in the recipient country.

- $\quad$ All donated drugs should be obtained from a reliable source and comply with quality standards in both the donor and recipient country.

- $\quad$ No drugs should be donated that have been issued to patients and then returned to a pharmacy or elsewhere, or were given to health professionals as free samples.

- After arrival in the recipient country all donated drugs should have a remaining shelf-life of at least one year.

- All drugs should be labelled in a language that is easily understood in the recipient country.

- $\quad$ As much as possible, donated drugs should be presented in larger quantity units and hospital packs.

For more information see the WHO document. 


\subsection{PROCUREMENT:}

The working group reviewed the current procurement systems of different organisations and evaluated the advantages and disadvantages of centralised and decentralised procurement as follows:

\section{CENTRALISED PROCUREMENT SYSTEM}

\begin{tabular}{|l|l|}
\hline ADVANTAGES & DISADVANTAGES \\
\hline - Low prices/Bulk purchase & - Limitation in suppliers' selection \\
- Quality assurance more feasible & - Security \\
- Adherence to suggested drug list & - Difficult to participate for those \\
- Improved planning and timely & organisations that run on short term \\
implementation & projects \\
- Less logistical and administrative problems & \\
- Appropriate centralised storage & \\
- Labelling and drug information in national & \\
languages & \\
- Facilitate Adverse Drug Reaction reporting & \\
- More possibility for research and studies & \\
- Assistance between organisations through & \\
drugs exchanges & \\
- Prevention of drug leakage to the market & \\
through putting a unique mark on packages & \\
\hline
\end{tabular}




\section{DECENTRALISED PROCUREMENT SYSTEM}

\begin{tabular}{|l|l|}
\hline \hline ADVANTAGES & DISADVANTAGES \\
\hline \hline - Freedom & - High prices \\
- Timing & - Quality assurance less feasible \\
& - Less adherence to suggested drug list \\
& - Difficulties in planning and timely \\
& implementation \\
& - More logistical and administerial problems \\
& - Problems in storage \\
& - Labelling and drug information in national \\
& languages is less feasible \\
& - Difficulty in Adverse Drug Reaction reporting \\
& - Less possibility for esearch and studies \\
& - Less chance for assistance between \\
& organisations through drugs exchanges \\
& - Prevention of drug leakage to the market is \\
& difficult, because authorities cannot differentiate \\
& easily donated drugs from others in the market \\
\hline
\end{tabular}

CONCLUSION: the working group suggests establishing a central procurement unit for joint purchasing. This system could be either established within a present organisation or a new one. If possible, the production of large-volume injectable solutions and Oral Rehidration Salts (ORS) should be supported at Avicena Pharmacutical Institute /MOPH, Kabul.

\subsection{QUALITY ASSURANCE:}

Taking into consideration of the importance of quality assurance the following reconmmendations are made:

- $\quad$ Reestablishment of the quality control laboratory in Kabul or within a wellfunctioning organisation.

- Quality control will be easier in a centralised procurement system. 
- WHO certification scheme on the quality of pharmaceutical products moving to international commerce should be used [5].

\subsection{STORAGE \& DISTRIBUTION:}

As several drugs require special storage conditions, special attention should be paid to this field.

- Improving the present stocks, as well as training store-keepers is recommended.

- Drugs procured by the aid community should carry a unique mark or symbol in order to be differentiated from other drugs available in the markets.

- In case of central procurement, problems with storage will decrease.

- Inter-organisational cooperation in drug storage especially at regional levels.

\subsection{DRUG USE:}

Drug use at the end point of drug utilisation has an important place. Irrational drug use can negate all the effort of drug supply.

\subsubsection{PRESCRIBERS:}

- $\quad$ Providing doctors with a copy of Afghan National Formulary [6]. The original document was published by MOPH and WHO.

- $\quad$ More topics on drug information for Mid-Level health workers through SALAMATY magazine.

- $\quad$ Preparing drug formulary for Mid-Level Health Workers e.g. shorter version of the above formulary, by MoPH and WHO.

- $\quad$ Organising training workshops for all levels of prescribers at medical institutions with technical assistance of WHO and UNICEF.

- $\quad$ Since all prescribed drugs are registered at facilities, studies on drug use should be encouraged by technical assistance of WHO and UNICEF.

- $\quad$ Special training workshops and research on drug use in Acute Respiratory Infections and in Diarrhoeal diseases with technical assistance of WHO and UNICEF.

(5) WHO certification scheme on the qulity of phannaceutical producst drugs moving to intenational conmerce. WHOPHARM. Geneva, February 1994.

(6) Afghan Naitioal Formulry, MOPH, 1996 (available form MOPH and WHO office, Afghanistan) 
- $\quad$ WHO and UNICEF offices in Afghanistan should be requested through ACBAR for new publications in the field of essential drugs.

- $\quad$ Restrictions should be made on use of opioids and psychotropic drugs. Dispense of opioids (Narcotics) like morphine, fentanyl, pentazocine (in the present list) as well as psychotropics like diazepam should be registered in a separate special file. This extra registration will help to control the misuse of said drugs.

- $\quad$ Over-use of injections should be prevented through training and information. The working group recommends all organisations to use disposable syringes only. This may appear to be more expensive, but providing fuel for sterilisation also requires funds and in addition sterilization is often done improperly.

\subsubsection{DISPENSING:}

- Qualified pharmacists/dispensers should be given priority for drug dispense

- Training workshops in community pharmacy should be organised for pharmacists and dispensers.

- Improving the communication between dispenser and patients at the health facilities.

\subsubsection{DRUG USE BY PATIENTS:}

- $\quad$ Public health education especially in the field of drug use through, mass media, schools and health facilities should be strengthen.

- Educational messages regarding rational drug use for patients should be formulated by a professional special committee. Messages should be prepared carefully taking into account the technical aspect as well as cultural and psychological factor and the knowledge of the patients. 


\section{APPENDIX I:}

\section{FURTHER READING:}

1- Afghan National Formulary, MOPH, 1996.

2- $\quad$ National list of Essential Drugs,MOPH, 1995.

3- $\quad$ Generic Law, MOPH, 1976.

4- The use of Essential Drugs, Model list of Essential Drugs, WHO,Geneva, 1994

5- $\quad$ Report on workshop on Rational use of Essential Drugs, UNICEF/Afghanistan Programme Office, 1996.

6- Collection of Hand-outs on Rational use of Essential Drugs,

UNICEF/Afghanistan Programme Office, 1996

7- The WHO Certification Scheme on the Quality of Pharmaceutical products Moving to International Commerce, WHO, Geneva, 1994.

8- Management Science for Health, International Drug Price Indicator Guide,MSH,Boston, Mass. 1993

9- $\quad$ UNICEF, Essential Drugs Price List, UNICEF, Copenhagen, 1996.

10- Guideline for developing national drug policy, WHO,Geneva, 1988.

11- Estimating Drug Requirement: a practical manual, WHO, Geneva, 1988.

12- $\quad$ Managing Drug Supply,MSH, Boston, Mass. 1985.

13- WHO, Conference of experts on the rational use of drugs. The rational use of drugs: report, WHO, Geneva 1987.

14- United Nations, Consolidated list of Products Whose Consumption and/or Sale Have Been Banned, Withdrawn ,Severely Restricted or not Approved by Governments, UN, New York, 1994. 


\section{APPENDIX II}

\section{DRUGS TO BE USED AT NATIONAL \& REGIONAL HOSPITALS}

\section{Anaesthetics}

\section{General anaesthetics and oxygen}

diazepam

halothane

ketamin

thiopental

oxygen

\section{Local anaesthetics}

lidocaine injection, $5 \mathrm{mg} / \mathrm{ml}$ in $2 \mathrm{ml}$ ampoule

inhalation

injection, $50 \mathrm{mg}$ (as hydrochloride) $/ \mathrm{mlin} 10 \mathrm{ml}$ vial powder for injection, $0.5 \mathrm{~g}, \mathrm{lg}$ (sodium salt) in ampoule inhalation (medicinal gas )

injection, $1 \%, 2 \%$ (hydrochloride) in vial injection $1 \%, 2 \%$ ( hydrochloride) + epinephrine $1 / 200000$ in vial

injection for spinal anaesthesia, $5 \%$ ( hydrochloride) in $2 \mathrm{ml}$ ampoule to be mixed with $7.5 \%$ glucose solution topical forms, $2 \%$ (hydrochloride)

injection, $1 \mathrm{mg}$ (sulfate) in $1 \mathrm{ml}$ ampoule

injection, $5 \mathrm{mg} / \mathrm{ml}$ in $2 \mathrm{ml}$ ampoule injection, $0.05 \mathrm{mg}$ (as citrate) $/ \mathrm{ml}$ in $2 \mathrm{ml}$ ampoule elixir, $5 \mathrm{mg}$ (hydrochloride) $/ 5 \mathrm{ml}$

2. Analgesics, antipyretics, non-steroid anti inflammatory drugs and drugs to treat gout

Non opioids

acetylsalicylic acid

allopurinol

colchicine

ibuprofen

indomethacin

paracetamol

\author{
tablet, 300, 500mg \\ tablet, $100 \mathrm{mg}$ \\ tablet, $0.5 \mathrm{mg}$ \\ tablet, $200 \mathrm{mg}$ \\ capsule, $25 \mathrm{mg}$ \\ tablet, $100,500 \mathrm{mg}$ \\ suppository, $100 \mathrm{mg}$ \\ syrup, $125 \mathrm{mg} / 5 \mathrm{ml}$
}

\section{Opioide analgesics}

To be used under restriction

Morphine(or pentazocine) injection, 10ng(sulfate) in $1 \mathrm{ml}$ ampoule 


\section{Antiallergics and drugs used in anaphylaxis}

chlorphenamine

dexamethasone

epinephrine

hydrocortisone

prednisolone tablet $4 \mathrm{mg}$ (hydrogen maleate)

injection $10 \mathrm{mg}$ (hydrogen maleate) in $1 \mathrm{ml}$ ampoule tablet $0.5 \mathrm{mg}, 4 \mathrm{mg}$ injection, $4 \mathrm{mg}$ (as sodium phosphate) in $1 \mathrm{ml}$ ampoule injection, $1 \mathrm{mg}$ (as hydrochloride) in $1 \mathrm{ml}$ ampoule powder for injection, 100mg(as sodium succinate)in vial tablet, $5 \mathrm{mg}$

\section{Antidote and other substances used in poisonings}

\section{General}

charcoal, activated

Specific

atropine

naloxone

\section{Antiepileptics}

carbamazepine

diazepam

ethosuximide

phenobarbital

phenytoin

valproic acid

\section{Anti-infective drugs}

\section{Anthelmintics \\ mebendazole \\ niclosamide \\ pyrentel}

\section{Antibacterial}

amoxicillin

ampicillin

benzathine benzyl penicillin

benzylpenicillin

cloxacillin

phenoxymethylpenicillin powder

injection, I mg (sulfăte) in I $\mathrm{ml}$ ampoule

injection, $0.4 \mathrm{mg}$ (hydrochloride) in $1 \mathrm{ml}$ ampoule

scored tablet, $100 \mathrm{mg}, 200 \mathrm{mg}$

injection, $5 \mathrm{mg} / \mathrm{ml}$ in $2 \mathrm{ml}$ ampoul (intravenous or rectal)

tablet, $250 \mathrm{mg}$

syrup $250 \mathrm{mg} / 5 \mathrm{ml}$

tablet, $15 \mathrm{mg}$ and $100 \mathrm{mg}$

elixir, $15 \mathrm{mg} / 5 \mathrm{ml}$

tablet, $25 \mathrm{mg}$ and $100 \mathrm{mg}$ (sodium salt)

enteric coated tablet,200nig(sodium salt) chewable tablet, $100 \mathrm{mg}$

chewable tablet, $500 \mathrm{mg}$

chewable tablet, $250 \mathrm{mg}$ (as embonate)

oral suspension $50 \mathrm{mg}$ (as embonate)/ml

capsule or tablet, $250 \mathrm{mg}, 500 \mathrm{mg}$ (anhydrous)

powder for oral suspension, $125 \mathrm{mg}$ (anhydrous) $/ 5 \mathrm{ml}$

powder for injection, $500 \mathrm{mg}$ (as sodium salt)in vial

powder for injection, $1.44 \mathrm{~g}$ benzylpenicillin $(=2.4$ million

IU)in vial

powder for injection , 2 million IU ( as sodium or potassium salt)

capsule, $500 \mathrm{mg}$ (as sodium salt )

powder for oral solution, $125 \mathrm{mg}$ (as sodium salt ) $/ \mathrm{ml}$

tablet, $2.50 \mathrm{mg}$ (as potassium salt)

powder for oral suspension, $250 \mathrm{mg}$ (as potassium salt) $/ 5 \mathrm{ml}$ 
procaine

benzyl penicillin

cliloramphenicol

erythromycin

gentamicin

metronidazole

sulfamethoxazole +

trimethoprime

tetracycline

ciprofloxacin

nitrofirantoin

ofloxacin powder for injection , I million IU

capsule,250mg, Oral suspension, 150mg (as

palmitate) salt $/ 5 \mathrm{ml}$

powder for injection, $1 \mathrm{~g}$ (as sodium succinate) in vial capsule or tablet, $250 \mathrm{mg}$ (as ethylsuccinate )

powder for oral suspension $125 \mathrm{mg}$ (as ethylsuccinate)

powder for injection, 500 $\mathrm{mg}$ (as lactobionate) in vial

injection, $40 \mathrm{mg}$ (as sulfate) $/ \mathrm{ml}$ in $2 \mathrm{nl}$ vial

injection, $500 \mathrm{mg}$ in $100 \mathrm{ml}$ vial

tablet, 100mig $+20 \mathrm{mg}, 400 \mathrm{mg}+80 \mathrm{mg}$

oral suspension, $200 \mathrm{mg}+40 \mathrm{mg} / 5 \mathrm{ml}$

capsule or tablet, $250 \mathrm{mg}$ (hydrochloride)

tablet, $250 \mathrm{mg}$ (as hydrochloride )

tablet, $100 \mathrm{mg}$

tablet, $200 \mathrm{mg}$

\section{Antituberculosis drugs}

(To be used only in the presence of anti-tuberculosis programmes)

ethambutol

isoniazid

pyrazinamide

Rifampicin

streptomycin

thiacetazone+isoniazid

\section{Antifungal drugs}

griseofulvin

ketoconazole

nystatin tablet, 400mg (hydrochloride )

tablet, 100mg, 300mg

tablet, 500mg

capsule or tablet $150 \mathrm{mg}, 300 \mathrm{mg}$

powder for injection, $\lg$ ( as sulfate) in vial

tablet, $50 \mathrm{mg}+100 \mathrm{mg} 150 \mathrm{mg}+300 \mathrm{mg}$

tablet, $125 \mathrm{mg}, 250 \mathrm{mg}$

tablet,200mg

oral suspension $100 \mathrm{mg} / 5 \mathrm{ml}$

tablet, 100000 IU,500 000IU

lozenge, $100000 \mathrm{IU}$

pessary, $100000 \mathrm{IU}$

\section{Antiprotozoal drugs}

Antiamoebic and antigiardiasis drugs

metronidazole

\section{Antimalarial drugs}

chloroquine

primaquine

quinine

sulfadoxine + pyrinethamine tablet,200mg,500mg oral suspension, $200 \mathrm{mg}$ (as benzozte)/5nl

tablet $150 \mathrm{mg}$ (as phosphate or sulfate) syrup, $50 \mathrm{mg}$ (as

phosphate or sulfate) $/ 5 \mathrm{ml}$

tablet, $7.5 \mathrm{mg}, 15 \mathrm{mg}$ (as diphosphate)

tablet,300mg (as bisulfate or sulfate)

injecition, $300 \mathrm{mg}$ (as dilyydrochloride) $/ \mathrm{ml}$ in $2 \mathrm{ml}$ ampoule

tablet, $500 \mathrm{mg}+25 \mathrm{mg}$ 


\section{Antimigraine drugs}

for treatment of acute attack.

acetylsalicylic acid

tablet.300, 500mg

ergotamine

tablet, $2 \mathrm{mg}$ (tartrate)

paracetamol

tablet. 500mg

for prophylaxis

propranolol

tablet. lomg

\section{Drugs affecting the blood}

\section{Antianaemia drugs}

ferrous salt +folic acid

hydroxocobalamin

tablet, 60m $+0.25 \mathrm{mg}$

iron dextran

injection, I mg in I 1 l anıpoule

injection, equivalent to $50 \mathrm{mg}$ iron/ml in $2 \mathrm{ml}$ ampoule

\section{Drugs affecting coagulation}

desmopressin

injection, 4 micro $\mathrm{g}$ (acetate) $/ \mathrm{ml}$ in I $\mathrm{ml}$ ampoule

heparin

phytonenadione

protamine sulfate injection, $1000 \mathrm{IU} / \mathrm{ml}, 5000 \mathrm{IU} / \mathrm{ml}, 20000 \mathrm{IU} / \mathrm{ml}$ in $\mathrm{I}-\mathrm{ml}$ ampoule

injection, $10 \mathrm{mg} / \mathrm{ml}$ in $5-\mathrm{ml}$ ampoule tablet, $10 \mathrm{mg}$

warfarin injection, $10 \mathrm{nig} / \mathrm{ml}$ in 5-ml ampoule tablet, $1 \mathrm{mg}, 2 \mathrm{mg}, 5 \mathrm{mg}$ (sodium salt)

\section{Blood product and plasma substitutes}

Plasma substitutes

dextran 70

polygeline

Plasma fraction for specific uses

albumin, human

factor VIII concentrate dried

(rarely used)

factor IX complex

(coagulation factors II, VII, IX,X)

concentrate

(rarely used)

\section{Cardiovascular drugs}

\section{Antianginal drugs}

glyceryl trinitrate injectable solution, $6 \%$

injectable solution, $3.5 \%$

injectable solution, $5 \%, 25 \%$

dried

tablet,(sublingual), $0.5 \mathrm{mg}$ 
isosorbide dinitrate

nifidipine

propranolol

atenolol

\section{Antidysrhythmic drugs}

lidocaine

propranolol

verapanil

atenolol

isoprenaline

procainamide

quinidine

Antihypertensive drugs

hydralazine

hydrochlorothiazide

nifidipine

propranolol

atenolol

methyldopa

sodium nitroprusside

Cardiac glycoside

digoxin

Drugs used in vascular shock

dopanine

\section{Antithrombotic drugs}

acetylsalicylic acid

streptokinase

\section{Dermatological drugs}

\section{Antifungal drugs (topical)}

benzoic acid+salicylic

acid

miconazole tablet.(sublingual), 5mg

capule and tablet, long

tablet, lomg

tablet, 100mg injection, $20 \mathrm{mg}$ (hydrochloride) $/ \mathrm{ml}$ in $5 \mathrm{ml}$ ampoule

tablet, 10mg, 40mg

injection. I mg (hydrochloride) in I $\mathrm{ml}$ ampoule

tablet, 40mg, $80 \mathrm{mg}$

injection, $2.5 \mathrm{mg}$ (hydrochloride ) $/ \mathrm{ml}$ in $2 \mathrm{ml}$ ampoule

tablet, 100mg

tablet, 10mg (hydrochloride)

injection, $0.2 \mathrm{mg}$ ((hydrochloride) $/ \mathrm{ml}$ in $2 \mathrm{ml}$ ampoule

tablet, $250 \mathrm{mg}, 500 \mathrm{mg}$ (hydrochloride)

tablet, $200 \mathrm{mg}$ (sulfate)

tablet, $25 \mathrm{mg}$, 50mg(hydrochloride) powder for injection, $20 \mathrm{mg}$ (hydrochloride) in ampoule

tablet, $25 \mathrm{~min}$

tablet, $10 \mathrm{mg}$

tablet. $40 \mathrm{mg}, 80 \mathrm{mg}$

tablet, $100 \mathrm{mg}$

tablet, $250 \mathrm{mg}$

powder for infusion, $50 \mathrm{mg}$ in ampoule

tablet, $0.25 \mathrm{mg}$

injection, $0.25 \mathrm{mg} / \mathrm{ml}$ in $2 \mathrm{ml}$ ampoule

injection, $40 \mathrm{mg}$ (hydrochloride) $/ \mathrm{ml}$ in $5 \mathrm{ml}$ vial

tablet, 100mg

powder for injection, 100000 IU in vial ointment, $6 \%+3 \%$

ointment, $2 \%$ ( nitrate ) 
Antiinfective drugs

metlyylrosanilinium

(gention violet)

neomycin + bacitracin

silver sulfadiazine aqueous solution, $0.5 \%$ chloride tincture, $0.5 \%$

ointment, 5 mg neomycin sulfate +500 IU bacitracin zinc / g cream, $1 \%$ in 500-g container

Anti-inflammatory and antipruritic drugs

betamethasone

calamine lotion

ointment, $0.1 \%$ (as valerate)

lotion

keratoplastic and keratolytic agents

coal tar

solution, topical $5 \%$

salicylic acid

solution, topical $5 \%$

Scabicides and pediculicides

benzyl benzoate

lotion , $25 \%$

Ultraviolet-blocking agents

zinc oxide

cream or ointment

\section{Diagnostic agents}

\section{Ophthalmic drugs}

fluorescin

eye drops, $1 \%$ (sodium salt)

(To be used in the presence of Ophthalmology Department)

\section{Radiocontrast media}

amidotrizoate

barium sulfate

iopamidol

ipanoic acid

propyliodone

meglumine iotroxate injection, $140 \mathrm{mg}$ iodine(as sodium or meglumine salts) $/ \mathrm{ml}$ in 20-ml ampoule aqueous suspension

aqueous solution, $370 \mathrm{mg}$ iodine $/ \mathrm{ml} \mathrm{in} 10 \mathrm{ml}$ ampoule tablet, $500 \mathrm{mg}$

oily suspension, $500 \mathrm{mg} / \mathrm{ml}$ in $20-\mathrm{ml}$ ampoule

injectable solution, $5 \mathrm{~g}$ iodine (as meglumine salt) in 100-250 ml

\section{Disinfectants and antiseptics}

\begin{abstract}
Antiseptics
chlorhexidine

hydrogen peroxide

polyvidone iodine

methylated spirit
\end{abstract}

\section{Disinfectants}

calcium hypochlorite powder, solution, $5 \%$ (diglugonate) for dilution

solution, $3 \%$

solution, containing $0.5 \%$ and $1 \%$ iodine

containing 5\% methyl alcohol and $95 \%$ ethyl alcohol

(70\% available chlorine) for solution 


\section{Diuretics}

furosemicle

hydrochlorothiazide

mannitol

spironolactone tablet, $40 \mathrm{mg}$

injection, $20 \mathrm{mg} / \mathrm{ml}$ in $2 \mathrm{ml}$ ampoule

tablet. $2.5 \mathrm{mg}, 50 \mathrm{mg}$

injectable solution, $10^{\circ}$ o, $20^{\circ}$ \%

tablet. $25 \mathrm{mg}$

\section{Gast rointestinal drugs}

Antacids and other antiulcer drugs

aluminium hydroxidet

tablet, 500nig

magnesium hydroxide

ranitidine

tablet, 150mg, 300mg

\section{Antienetic drugs}

metoclopranide

tablet, 10mg (as hydrochloride)

oral solution, $5 \mathrm{mg}$ (as hydrochloride) $5 / \mathrm{ml}$

injection, $5 \mathrm{mg}$ (as hydrochloride) $/ \mathrm{nl}$ in $2 \mathrm{ml}$ anıoule

promethazine

tablet, 10mg, 25mg (hydrochloride)

syrup, $5 \mathrm{nig}$ (hydrochloride) $/ \mathrm{ml}$ injection,

$25 \mathrm{mg}$ (hydrochloride) $/ \mathrm{ml}$ in $2 \mathrm{ml}$ anpoule

Antihaemorrhoidal drugs

local anaesthetic,

ointment or suppository astringent and anti-inflammatory drugs

\section{Antispasmodic drugs}

hyoscine

tablet, 10mg (butylbromide)

injection, $4 \mathrm{mg}$ (butylbromide) $/ \mathrm{ml}$ in $5 \mathrm{ml}$ ampoule

Compound preparations of hyoscine are not advised.

\section{Cathartic drugs}

senna

tablet, $75 \mathrm{mg}$ (sennosides)

Drugs used in diarrhoea

Oral reliydration

oral rehydration salts

powder, $27.9 \mathrm{~g} / 1$

('omponents

sodium chloride

trisodiun citrate dihydrate

potassium chloride

glucose g litre'

3.5

2.9

1.5

20 
16. Hormones, other endocrine drugs

Adrenal hormones and synthetic substitutes

dexamethasone

tablet, $0.5 \mathrm{mg}, 4 \mathrm{mg}$

injection, $4 \mathrm{mg}$ (as sodium phosphate) in $1-\mathrm{ml}$ ampoule

hydrocortisone

powder for injection, $100 \mathrm{mg}$ (as sodium succinate) in vial

prednisolone

tablet. $1 \mathrm{mg}, 5 \mathrm{mg}$

\section{Estrogen}

ethinylestradiol

tablet, $0.05 \mathrm{mg}$

\section{Insulins and other antidiabetic agents}

insulin

injection, $40 \mathrm{IU} / \mathrm{ml}$ in $10 \mathrm{ml}$ vial. injection (soluble) $80 \mathrm{IU} / \mathrm{ml}$ in 10ull vial

intermediate acting

injection, $40 \mathrm{lU} / \mathrm{ml}$ in $10 \mathrm{ml}$ vial, insulin $80 \mathrm{IU} / \mathrm{ml}$ in $10-\mathrm{ml}$ vial(as compound insulin zinc suspension or isophane insulin)

tolbutamide tablet, 500ming

metformin tablet, 500mg (hydrochloride)

\section{Progesterons}

norethisterone

tablet, $5 \mathrm{mg}$

\section{Thyroid hormones and antithyroid drugs}

levothyroxine

potassium iodide

propylthiouracil

\section{7.lmmunologicals}

\section{Diagnostic agents}

tuberculin, purified tablet, $0.05 \mathrm{mg}, 0.1 \mathrm{mg}$ (sodium salt)

tablet, $60 \mathrm{mg}$

tablet, $50 \mathrm{mg}$

(To be used in the presence of Anti-tubercluosis programmes)

\section{Immunglobulins}

antitetanus

anti rabies vaccine

injection immunglobulin (human)

injection

Vaccines supply should be in line with national Extended Programme of Immunisation (EPI).

\section{Muscle relaxants(peripherally acting) and anticholinesterase}

neostignine

pancuronium

suxamethonium tablet, I5 mg(bromide)

injection, $0.5 \mathrm{mg}, 2.5 \mathrm{mg}$ (metilsulfate) in $1-\mathrm{ml}$ ampoule injection, $2 \mathrm{mg}$ (bromide) $/ \mathrm{ml}$ in $2 \mathrm{ml}$ ampoule injection, $50 \mathrm{mg}$ (chloride) $/ \mathrm{ml}$ in 2-ml ampoule powder for injection, (chloride) 


\section{Ophthalnological preparations}

Anti-infective agents

gentamicin

silver nitrate

tetracycline solution, (eye drops), $0.3 \%$

solution.(eye drops), $1 \%$

eye ointment $1 \%$, (hydrochloride)

\section{Anti-inflammatory agents}

(To be used in the presence of Ophthalmology Department)

prednisolone

solution, (eye drops), $0.5 \%$

\section{Local anaestlietics}

(To be used in the presence of Ophthalmology Department)

tetracaine

solution(eye drops), $0.5 \%$ (hydrochloride)

Miotics and antiglucoma dr'ugs

(To be used in the presence of Ophthalmology Department)

acetazolanide

pilocarpine tablet, $250 \mathrm{mg}$

solution,(eye drops), $2 \%, 4 \%$

(hydrochloride or nitrate)

\section{Mydriatics}

(To be used in the presence of Ophthalmology Department)

atropine

solution(eye drops), $0.1 \% .0 .5 \%, 1 \%$ (sulfate)

\section{Oxytocics and antioxitocics}

\section{Oxytocics}

ergometrine

oxytocine

\section{Antioxytocics}

salbutamol

\section{Psychotherapeutics drugs}

amitriptylline

chlorpromazine

diazepam tablet, $0.2 \mathrm{mg}$ (hydrogen maleate) injection, $0.2 \mathrm{mg}$ (hydrogen maleate) in $1-\mathrm{ml}$ ampoule injection, $10 \mathrm{IU}$ in $1 \mathrm{ml}$ ampoule

tablet, $4 \mathrm{mg}$ (as sulfate)

tablet, $25 \mathrm{mg}$ (hydrochloride)

tablet, $100 \mathrm{mg}$, (hydrochloride)

syrup, $25 \mathrm{mg}$ (hydrochloride) $/ 5 \mathrm{ml}$

injection, $25 \mathrm{mg}$ (hydrochloride)/ $\mathrm{ml}$ in 2-ml ampoule tablet, $2 \mathrm{mg}$, $5 \mathrm{mg}$ 


\section{Drug acting on the respiratory tract}

\section{Antiasthmatic drugs}

aminophylline

tablet , 100mg, 200nig

injection, $25 \mathrm{mg} / \mathrm{ml}$ in $10 \mathrm{ml}$ ampoule

ejincphrine

salbutamol

injection, Ing (as hydrochloride) in $1 \mathrm{ml}$ ampoule

tablet, $2 \mathrm{mg}$, $4 \mathrm{mg}$ (as sulfate)

inhalation, $0.1 \mathrm{mg}$ (as sulfate) per dose

syrup, $2 \mathrm{mg}$ (as sulfate) $/ 5 \mathrm{ml}$

injection, $0.05 \mathrm{mg}$ (as sulfate) $/ \mathrm{mll}$ in $5 \mathrm{nll}$ anıpoule

\section{Antitussives}

dextromethorphan

syrup, $15 \mathrm{mg}$ (hydrobromide)/5nl

\section{Solutions correcting water, electrolyte and acid-base disturbances}

\section{Oral reliydration}

oral relydration salts

(

sodium chloride

trisodium citrate diliydrate

potassium chloride

glucose

potassium chloride

\section{Parenteral}

aminoacids

glucose

glucose with

sodiunı chloride

potassium cliloride

sodium chloride

sodium hydrogen

carbonate

(sodium bicarbonate)

compound solution of

sodiun lactate

$$
\text { powder, } 27.9 \mathrm{~g} / 1
$$

y lime

3.5

2.9

1.5

20

powder for solution

injectable solution $3.5 \%$

injectable solution, $5 \%$ isotonic, $25 \%$ hypertonic

injectable solution, $4 \%$ glucose,

$0.18 \%$ sodiun chloride (equivalent to $\mathrm{Na} 30 \mathrm{mmol} / \mathrm{ml}$,

$\mathrm{Cl}^{-} 30 \mathrm{mmol} / 1$

$11.2 \%$ solution in $20 \mathrm{ml}$ ampoule (equivalent to $\mathrm{K} .5 \mathrm{mmol} / \mathrm{ml}$ $\mathrm{Cl}^{-} 1.5 \mathrm{mmol} / \mathrm{ml}$ )

injectable solution, $0.9 \%$ isotonic (equivalent to $\mathrm{Na} 154 \mathrm{mmol} / \mathrm{l}$, $\mathrm{Cl}^{-}$154mnol/l)

injectable solution, $1.4 \%$ isotonic (equivalent to $\mathrm{Na}$. $\left.167 \mathrm{mmol} / 1, \mathrm{HCO}^{-3} 167 \mathrm{mmol} / \mathrm{l}\right)$

$8.4 \%$ solution in $10 \mathrm{ml}$ ampoule (equivalent to $\mathrm{Na}$

$\left.\mathrm{mol} / \mathrm{l}, \mathrm{HCO}^{-3} 1 \mathrm{~mol} / \mathrm{l}\right)$

injectable solution

\section{Miscellaneous}

water for injection

$2 \mathrm{nll}, 5 \mathrm{ml}$ ampoule 


\section{Vitamins and minerals}

ascorbic acid

calcium

ergoclciferol

iodine

nicotinamide

pyridoxine

retinol

ribofavin

sodium floride

thiamine tablet, $50 \mathrm{mg}$

tablet. 500mg(lactate)

injection $100 \mathrm{mg}$ (gluconate)/ $\mathrm{ml}$ in $10 \mathrm{ml}$ ampoule

capsule or tablet, $1.25 \mathrm{mg}$ (50 $000 \mathrm{IU}$ )

oral solution, $0.25 \mathrm{mg} / \mathrm{ml}(10000 \mathrm{IU} / \mathrm{ml})$

iodized oil, $1 \mathrm{ml}$ (480mg iodine), $0.5 \mathrm{ml}(240 \mathrm{mg}$ iodine) in ampoule (oral or injectable)

capsule, $200 \mathrm{mg}$

tablet $50 \mathrm{mg}$

tablet, $25 \mathrm{mg}$ (hydrochloride)

sugar coated tablet, $10000 \mathrm{IU}$ ( as palmitate) $(5.5 \mathrm{mg}$ )

capsule, $200000 \mathrm{IU}$ ( as palnitate)(110 mg)

oral oily solution, $100000 \mathrm{IU} / \mathrm{ml}$ in multidose dispenser( as palmitate)

water-miscible injection, 100 000IU ( as palmitate) $(55 \mathrm{mg})$ in $2 \mathrm{ml}$

tablet, $5 \mathrm{mg}$

tablet $0.5 \mathrm{mg}$

solution, $2 \%$ (for professional dental use)

tablet, $50 \mathrm{mg}$ (hydrochloride)

\section{REMARKS}

At present no NGO is involved in the treatment of Leprosy, Cancer, Parkinson and Leishmania Drugs to treat these diseases was not disscused here. For interrested organisations in those drugs, reference is made to National List of Essential Drugs, MOPH, 1995, that is available from MOPH and WHO 


\section{DRUGS TO BE USED AT PROVENCIAL \& DISTRICI HOSPITALS}

\section{Anaesthetics}

General anaesthetics and oxygen diazepam

ketamin

thiopenthal

oxygen

\section{local anaesthetics}

lidocaine injection, $5 \mathrm{mg} / \mathrm{ml}$ in $2 \mathrm{nl}$ ampoule

injection, 50mg(ashydrochloride)/ $/ \mathrm{ml}$ in $10 \mathrm{nl}$ vial

powder for injection, $0.5 \mathrm{~g}$ and $1 \mathrm{~g}$ (sodium salt ) in ampoule inhalation (medicinal gas)

injection. $1^{\circ} \%, 2 \%$ (hydrochloride) in vial

injection $1 \% .2 \%$ ( hydrochloride) + epinephrine $1 / 200000$ in vial injection for spinal anaesthesia, $5 \%$ (hydrochloride) in $2 \mathrm{ml}$ anpoule to be mixed with $7.5 \%$ glucose solution topical forms, $2 \%$ (hydrochloride)

injection, I $\mathrm{mg}$ (sulfate) in $\mathrm{lml}$ ampoule

injection, $5 \mathrm{nig} / \mathrm{ml}$ in $2 \mathrm{ml}$ ampoule

elixir, $5 \mathrm{mg}$ (hydrochloride) $/ 5 \mathrm{ml}$

\section{Analgesics, antipyretics, non-steroid anti inflammatory drugs and drugs to treat gout}

\section{Non opioids \\ acetylsalicylic acid \\ ibuprofen \\ indomethacin \\ paracetamol}

\section{Opioide analgesics}

morphine

\author{
tablet, 300, 500mg \\ tablet, 200my \\ capsule, $25 \mathrm{mg}$ \\ tablet, 100,500mg \\ suppository, 100mg \\ syrup, $125 \mathrm{mg} / 5 \mathrm{ml}$
}

injection, 10mg(sulfate) in $1 \mathrm{ml}$ ampoule (OR pentazocine)

(To be used under restriction)

\section{Antiallergics and drugs used in anaphylaxis}

chlorphenamine

epinephrine

hydrocortisone

prednisolone tablet $4 \mathrm{mg}$ (hydrogen maleate)

injection 10nig (hydrogen maleate) in I nl ampoule injection, $1 \mathrm{mg}$ (as hydrochloride) in $1 \mathrm{ml}$ ampoule powder for injection, 100mg(as sodium succinate)in vial tablet, $5 \mathrm{mlg}$ 


\section{Antidote and other substances used in poisonings}

\section{General}

charcoal. activated

\section{Specific}

atropine

naloxone

powder

injection, I mig (sulfate) in I $\mathrm{ml}$ ampoule

injection, $0.4 \mathrm{mg}$ (hydrochloride) in $\mathrm{lml}$ ampoule

\section{Antiepileptics}

diazepam

Injection, $5 \mathrm{mg} / \mathrm{ml}$ in $2 \mathrm{ml}$ ampoule

ethosuximide

(intravenous or rectal)

tablet, 250ning

syrup $250 \mathrm{mg} / 5 \mathrm{ml}$

phenobarbital

tablet, $15 \mathrm{mg}$ and $100 \mathrm{mg}$

elixir, $15 \mathrm{mg} / 5 \mathrm{ml}$

phenytoin

tablet.25mg and 100ng (sodium salt)

\section{Anti-infective drugs}

\section{Anthelmintics}

niclosamide

pyrentel

\section{Antibacterial}

amoxicillin

ampicillin

benzathine benzyl penicillin

benzylpenicillin

cloxacillin

phenoxymethylpenicillin

procaine

benzylpenicillin

chloramphenicol

(To be used under restriction)

erythromycin

metronidazole

sulfamethoxazole +trimethoprime

tetracycline

nitrofurantoin

chewable tablet, $500 \mathrm{mg}$

chewable tablet, $250 \mathrm{mg}$ (as embonate)

oral suspension $50 \mathrm{mg}$ (as embonate)/nl

capsule or tablet,250nig, 500mg (anhydrous)

powder for oral suspension, $125 \mathrm{mig}$ (anhydrous) $/ 5 \mathrm{ml}$

powder for injection, $500 \mathrm{mg}$ (as sodium salt)in vial

powder for injection, $1.44 \mathrm{~g}$ benzylpenicillin $(=2.4$ million

IU)in vial

powder for injection ,2 million IU ( as sodium or potassium salt)

capsule, $500 \mathrm{mg}$ (as sodium salt )

powder for oral solution, $125 \mathrm{mg}$ (as sodium salt )/ml

tablet, $250 \mathrm{mg}$ (as potassium salt)

powder for oral suspension, $250 \mathrm{mg}$ (as potassium salt) $/ 5 \mathrm{ml}$

powder for injection , 1 million IU

capsule, $250 \mathrm{~m}$ g oral suspension, $150 \mathrm{mg}$ (as palmitate salt) $/ 5 \mathrm{ml}$ powder for injection, $\mathrm{g}$ (as sodium succinate) in vial capsule or tablet, $250 \mathrm{mg}$ (as ethylsuccinate ) powder for oral use, $125 \mathrm{mg}$ (as ethylsuccinate) $/ 5 \mathrm{ml}$ gentamicin injection, $40 \mathrm{mg}$ (as sulfate) $/ \mathrm{ml}$ in $2 \mathrm{ml}$ vial

tablet, $200,500 \mathrm{mg}$ injection, $500 \mathrm{mg}$ in $100 \mathrm{ml}$ vial

tablet, $100 \mathrm{mg}+20 \mathrm{mg}, 400 \mathrm{mg}+80 \mathrm{mg}$ oral suspension, $200 \mathrm{mg}+40 \mathrm{mg} / 5 \mathrm{ml}$ capsule or tablet, $250 \mathrm{mg}$ (hydrochloride) tablet, $100 \mathrm{mig}$ 


\section{Anituberculosis drugs}

(To be used in the presence of Anti-tuberculosis programmes)

ethambutol

isoniazid

pyrazinamide

Rifampicin

streptomycin

thiacetazone+isoniazid

Antifungal drugs

griseofulvin

nystatin tablet, 400mg (hydrochloride )

tablet, 100mg, 300mg

tablet, 500mg

capsule or tablet $150 \mathrm{mg}, 300 \mathrm{mg}$

powder for injection, $\mathrm{Ig}$ ( as sulfate)in vial

tablet, $50 \mathrm{mg}+100 \mathrm{mg} 150 \mathrm{mg}+300 \mathrm{mg}$

tablet, 125mg, 250mg

tablet. 100000 IU,500 000IU

lozenge, $100000 \mathrm{IU}$

pessary, $100000 \mathrm{IU}$

\section{Antiprotozoal drugs}

Antiamoebic and antigiardiasis drugs

metronidazole

tablet,200nig,500mg

injection, $500 \mathrm{mg}$ in $100 \mathrm{nl}$ vial

oral suspension, $200 \mathrm{mg}$ (as benzozte) $/ 5 \mathrm{ml}$

\section{Antinualarial drugs}

chloroquine

tablet $150 \mathrm{mg}$ (as phosphate or sulfate)

prinlacuine

syrup,50mg(as phosphate or sulfate) $/ 5 \mathrm{ml}$

quinine

tablet, $7.5 \mathrm{mg}, 15 \mathrm{mg}$ (as diphosphate)

tablet, $300 \mathrm{mg}$ (as bisulfate or sulfate)

injecition, 300mg (as dihydrochloride) $/ \mathrm{ml}$ in $2 \mathrm{ml}$ ampoule

sulfadoxine + pyrimethanine tablet, $500 \mathrm{mg}+25 \mathrm{mg}$

\section{Antimigraine drugs}

\section{for treatment of acute attack}

acetylsalicylic acid

tablet, 300, 500mg

ergotamine

tablet, $2 \mathrm{mg}$ (tartrate)

paracetamol

tablet, 500mg

\section{for prophylaxis}

propranolol

tablet, 10mg

\section{Drugs affecting the blood}

\section{Antianaemia drugs}

ferrous salt+folic acid

hydroxocobalamin

tablet, $60 \mathrm{n} / \mathrm{g}+0.25 \mathrm{mg}$

injection, $1 \mathrm{mg}$ in $1 \mathrm{ml}$ ampoule 


\section{Drugs affecting coagulation}

(To be used in the presence of Cardiovascular department)

heparin

phytomenadione

protamine sulfate injection, $1000 \mathrm{IU} / \mathrm{ml}, 5000 \mathrm{IU} / \mathrm{ml}, 20000 \mathrm{IU} / \mathrm{ml}$ in $1-\mathrm{ml}$ ampoule

injection, $10 \mathrm{mg} / \mathrm{ml}$ in $5-\mathrm{ml}$ ampoule

tablet, $10 \mathrm{mg}$

injection, $10 \mathrm{mg} / \mathrm{ml}$ in $5-\mathrm{ml}$ ampoule

\section{Blood product and plasma substitutes}

\section{Plasmal substitutes}

dextran 70

Plasma fraction for specific uses

albunin, human

\section{Cardiovascular drugs}

\section{Antianginal drugs}

glyceryl trinitrate

isosorbide dinitrate

nifidipine

propranolol

\section{Antidysrhythmic drugs}

propranolol

\section{Antiliypertensive drugs}

hydrochlorothiazide

nifidipine

Methyl doapa

\section{Cardiac glycoside}

digoxin injectable solution, $6 \%$

injectable solution, $5 \%, 25 \%$ tablet,(sublingual), $0.5 \mathrm{mg}$

tablet,(sublingual), $5 \mathrm{mg}$

tablet, 10mg

tablet, 10mg

tablet, $10 \mathrm{mg}, 40 \mathrm{mg}$

injection, I Img (hydrochloride) in Inl ampoule

tablet, $25 \mathrm{mg}$ tablet, $10 \mathrm{mg}$

tablet, $250 \mathrm{mg}$

tablet, $0.25 \mathrm{mg}$

oral solution, $0.05 \mathrm{mg} / \mathrm{ml}$

injection, $0.25 \mathrm{mg} / \mathrm{ml}$ in $2 \mathrm{ml}$ ampoule

injection, $40 \mathrm{mg}$ (hydrochloride) $/ \mathrm{ml}$ in $5 \mathrm{ml}$ vial

Drugs used
dopamine

Antitlurombotic drugs

acetylsalicylic acid

tablet,300mg 


\section{Dermatological drugs}

Antifungal drugs (topical)

benzoic acid+salicylic

ointment, $6 \%+3 \%$

acid

miconazole

ointment. $2 \%$ ( nitrate )

Antiinfective drugs

methylrosanilinium

(gention violet)

neomycin + bacitracin

aqueous solution, $0.5 \%$ chloride tincture, $0.5 \%$

ointment, $5 \mathrm{mg}$ neomycin sulfate +

$500 \mathrm{IU}$ bacitracin zinc / $\mathrm{g}$

silver sulfadiazine

cream. $1 \%$ in 500-g container

Anti-inflamınatory and antipruritic drugs

calamine lotion

lotion

Bethanethazon

ointment,

keratoplastic and keratolytic agents

coal tar

salicylic acid

solution, topical $5 \%$

solution, topical $5 \%$

\section{Scabicides and pediculicides}

benzyl benzoate

zinc oxide

lotion, $25 \%$

cream or ointment

\section{Diagnostic agents}

\section{Radiocontrast media}

anidotrizoate

barium sulfate

injection, $140 \mathrm{mg}$ iodine(as sodium or meglumine salts) $/ \mathrm{ml}$ in 20-ml ampoule

aqueous suspension

\section{Disinfectants and antiseptics}

\section{Antiseptics}

chlorhexidine

lydrogen peroxide

polyvidone iodine

methylated spirit

solution, $5 \%$ (digluconate) for dilution

solution, $3 \%$

solution, containing $0.5 \%$ and $1 \%$ iodine

containing 5\% methyl alcohol and 95\% ethyl alcohol

\section{Disinfectants}

calcium hypochlorite

powder, ( $70 \%$ available chlorine) for solution

\section{Diuretics}

furosemide

tablet, $40 \mathrm{mg}$

injection, $20 \mathrm{mg} / \mathrm{ml}$ in $2 \mathrm{ml}$ ampoule

hydrochlorothiazide

tablet, $25 \mathrm{mg}, 50 \mathrm{mg}$

mannitol

injectable solution, $10 \%, 20 \%$ 


\section{Gastrointestinal drugs}

Antacids and other antinlcer drugs

aluminium hydroxide+

tablet, 500ng

magnesium hydroxide

ranitidine

tablet, 150mg, 300mg

Antiemetic drugs

metoclopramide

tablet, 10mg (as hydrochloride)

oral solution, $5 \mathrm{mg}$ (as hydrochloride) $5 / \mathrm{ml}$

injection, $5 \mathrm{mg}$ (as hydrochloride)/ $\mathrm{ml}$ in $2 \mathrm{ml}$ ampoule

promethaine tablet, 10mg. $25 \mathrm{mg}$ (hydrochloride)

syrup, $5 \mathrm{mg}$ (hydrochloride)/ml

injection, $25 \mathrm{mg}$ (hydrochloride)/nl in 2-ml ampoule

\section{Antihaemorrhoidal drugs}

local anaesthetic,

ointment or suppository astringent and anti-inflammatory drugs

Antispasmodic drugs

hyoscine

tablet, 10mig (butylbromide)

injection, $4 \mathrm{mg}$ (butylbromide) $/ \mathrm{ml}$ in $5 \mathrm{ml}$ ampoule

( Compound preparation of hyoscine are not advised)

Cathartic drugs

senna

tablet, 7.5mg(sennosides)

Drugs used in diarrhoea

Oral rehydration

oral rehydration salts

('omponents

powder, $27.9 \mathrm{~g} / \mathrm{l}$

sodium chloride

$g$ lirre

trisodium citrate dihydrate

3.5

potassium chloride

glucose

16. Hormones, other endocrine drugs

Adrenal hormones and synthetic substitutes

hydrocortisone

powder for injection, $100 \mathrm{mg}$ (as sodium

succinate) in vial

prednisolone

tablet, $1 \mathrm{mg}, 5 \mathrm{mg}$ 
Insulins and other antidiabetic agents

insulin

intermediate acting

insulin

metformin

\section{Progesterons}

norethisterone injection, $40 \mathrm{IU} / \mathrm{ml}$ in $10 \mathrm{ml}$ vial, injection(soluble) $80 \mathrm{IU} / \mathrm{ml}$ in $800 \mathrm{ml}$ vial

injection, $40 \mathrm{IU} / \mathrm{ml}$ in $10 \mathrm{ml}$ vial,

$80 \mathrm{IU} / \mathrm{ml}$ in 10-ml vial(as compound insulin zinc suspension or isophane insulin)

tablet,

tablet, $5 \mathrm{mg}$

Thyroid hornones and antitlyyroid drugs

( to be used in endemic area)

levothyroxine

potassium iodide

propylthiouracil tablet, $0.05 \mathrm{mg}, 0.1 \mathrm{mg}$ (sodium salt)

tablet, $60 \mathrm{mg}$

tablet, $50 \mathrm{mg}$

\section{7.immunologicals}

\section{Diagnostic agents}

tuberculin, purified

injection protein derivative (PPD)

(To be used in the presence of Anti-tubercluosis programmes)

\section{Immunglobulins}

antitetanus

injection immunglobulin (human)

Vaccines should be in line with national Extended Programme of Immunisation (EPI).

18. Muscle relaxants(peripherally acting) and

anticholinesterase

neostigmine

tablet, $15 \mathrm{mg}$ (bromide)

injection, $0.5 \mathrm{mg}, 2.5 \mathrm{mg}$ (metilsulfate) in $1-\mathrm{ml}$ ampoule

pancuronium injection, $2 \mathrm{mg}$ (bromide) $/ \mathrm{ml}$ in $2 \mathrm{ml}$ ampoule

suxamethonium injection, $50 \mathrm{mg}$ (chloride) $/ \mathrm{ml}$ in $2-\mathrm{ml}$ ampoule powder for injection, (chloride)

\section{Ophthalnıological preparations}

Anti-infective agents

gentamicin

solution,(eye drops), $0.3 \%$

silver nitrate

solution,(eye drops), $1 \%$

tetracycline

eye ointment $1 \%$, (hydrochloride)

\section{Anti-inflammatory agents}

(To be used in Ophthalmology department)

prednisolone

solution,(eye drops), $0.5 \%$ 
Miotics and antiglucoma drugs

(To be used in Ophthalmology department)

acetazolamide

tablet, $250 \mathrm{mg}$

pilocarpine solution,(eye drops), $2 \%, 4 \%$

(hydrochloride or nitrate)

\section{Mydriatics}

(To be used in Ophthalmology department)

atropine

solution(eye drops), $0.1 \%, 0.5 \%, 1 \%$ (sulfate)

20 Oxytocics and antioxytocics

Oxytocics

ergometrine

tablet, $0.2 \mathrm{mg}$ (hydrogen maleate)

injection, $0.2 \mathrm{mg}$ (hydrogen maleate) in 1 -ml ampoule oxytocine injection, $10 \mathrm{IU}$ in $1 \mathrm{ml}$ ampoule

\section{Antioxytocics}

salbutamol

tablet, $4 \mathrm{mg}$ (as sulfate)

\section{Psychotherapeutics drugs}

anitriptylline

tablet, $25 \mathrm{mg}$ (hydrochloride)

chlorpromazine

tablet, $100 \mathrm{mg}$, (hydrochloride)

syrup, $25 \mathrm{mg}$ (hydrochloride) $/ 5 \mathrm{ml}$

injection, $25 \mathrm{mg}$ (hydrochloride) $/ \mathrm{ml}$ in 2-ml ampoule

diazepam tablet, $2 \mathrm{mg}$, $5 \mathrm{mg}$

22 Drug acting on the respiratory tract

\section{Antiasthmatic drugs}

aminophylline

epinephrine

salbutanıol

tablet , 100mg,200mg

injection, $25 \mathrm{mg} / \mathrm{ml}$ in $10 \mathrm{ml}$ ampoule

injection, I $\mathrm{mg}$ (as hydrochloride) in $1 \mathrm{ml}$ ampoule

tablet, $2 \mathrm{mg}$, $4 \mathrm{mg}$ (as sulfate)

inhalation, $0.1 \mathrm{mg}$ (as sulfate) per dose

syrup, $2 \mathrm{mg}$ (as sulfate) $/ 5 \mathrm{ml}$

injection, $0.05 \mathrm{mg}$ (as sulfate) $/ \mathrm{ml}$ in $5 \mathrm{ml}$ ampoule

\section{Antitussives}

dextromethorphan

syrup, $15 \mathrm{mg}$ (hydrobromide)/5ml 
23. Solutions correcting water, electrolyte and acid-base disturbances

Oral rehydration

oral relhydration salts

$($ (im) (m)

sodium chloride

trisodium citrate dihydrate

potassium chloride

glucose

potassium chloride

\section{Parenteral}

glucose

glucose with

sodium chloride

sodium chloride

sodiun hydrogen

carbonate

(sodium bicarbonate)

compound solution of

sodium lactate

\section{Miscellaneous}

water for injection

\section{Vitamins and minerals}

ascorbic acid

calcium glugonate

calcium

ergoclciferol

iodine

nicotinamide

pyridoxine

retinol powder, $27.9 \mathrm{~g} / 1$

g litre

3.5

2.9

1.5

20

powder for solution

injectable solution, $5 \%$ isotonic, $25 \%$ hypertonic injectable solution, $4 \%$ glucose,

$0.18 \%$ sodium chloride (equivalent to $\mathrm{Na} 30 \mathrm{mmol} / \mathrm{ml}, \mathrm{Cl}^{-}$ $30 \mathrm{mmol} / \mathrm{l}$

injectable solution, $0.9 \%$ isotonic

(equivalent to $\mathrm{Na} 154 \mathrm{mmol} / 1, \mathrm{Cl}^{-} 154 \mathrm{mmol} / \mathrm{l}$ )

injectable solution, $1.4 \%$ isotonic(equivalent

to $\left.\mathrm{Na}^{\cdot} 167 \mathrm{mmol} / \mathrm{l}, \mathrm{HCO}^{-} 167 \mathrm{mmol} / \mathrm{l}\right)$

$8.4 \%$ solution in $10 \mathrm{ml}$ anpoule (equivalent to $\mathrm{Na} 1 \mathrm{~mol} / \mathrm{l}$, $\mathrm{HCOO} 3^{-}$I $\left.\mathrm{mol} / \mathrm{l}\right)$

injectable solution

$2 \mathrm{ml}, 5 \mathrm{ml}$ ampoule

tablet, $50 \mathrm{mg}$

injection $100 \mathrm{mg} / \mathrm{ml}$ in $10 \mathrm{ml}$ ampoule

tablet, 500mg(lactate)

capsule or tablet, $1.25 \mathrm{mg}$ (50 $000 \mathrm{IU}$ ) oral solution, $0.25 \mathrm{mg}$ $/ \mathrm{ml}(10000 \mathrm{IU} / \mathrm{ml})$

iodized oil, $1 \mathrm{ml}$ (480mg iodine), $0.5 \mathrm{ml}(240 \mathrm{n} \mathrm{g}$ iodine) in asmpoule (oral or injectable) capsule, $200 \mathrm{mg}$

tablet $50 \mathrm{nig}$

tablet, 25 nig (hydrochloride)

sugar coated tablet, $10000 \mathrm{IU}$ ( as palmitate) (5.5mg)

capsule, $200000 \mathrm{IU}$ ( as palmitate)(110mg)

oral oily solution, $100000 \mathrm{IU} / \mathrm{ml}$ in multidose dispenser( as palmitate)

water-miscible injection, 100 000IU (as palmitate) $(55 \mathrm{mg})$ in $2 \mathrm{ml}$ anpoule 
ribofavin

sodium floride

thiamine tablet, $5 \mathrm{mg}$ tablet, $0.5 \mathrm{mg}$

solution, $2 \%$ (for professional dental use)

tablet, $50 \mathrm{mg}$ (hydrochloride) 
(Prescribers: doctors)

\section{Anaesthetics}

Local anaesthetics

lidocaine

injection, $1 \% .2 \%$ (hydrochloride) in vial

injection $1 \%, 2 \%$ ( hydrochloride) + epinephrine $1 / 200000$

in vial

topical forms. $2 \%$ (hydrochloride)

2. Analgesics, antipyretics, non-steroid anti inflammatory dhugs and dnugs to treat gont

acetylsalicylic acid

ibuprofen

paracetamol

morpline tablet, 100, 500nig

tablet, 200mg

tablet, 100,500mg

suppository, $100 \mathrm{mg}$

syrup, $125 \mathrm{mg} / 5 \mathrm{ml}$

injection, 10mg(sulfate) in $1 \mathrm{ml}$ ampoule (OR pentazocine)

(lo be used under restriction)

\section{Antiallergics and drugs used in anaphylaxis}

chlorphenamine

epinephrine

hydrocortisone tablet 4 nig (hydrogen maleate)

injection 10mg (hydrogen maleate) in $1 \mathrm{ml}$ ampoule injection, I mg (as hydrochloride) in $1 \mathrm{ml}$ anipoule powder for injection, $100 \mathrm{mg}$ (as sodium succinate) in vial

\section{Antidote and other substances nsed in poisonings}

\section{General}

charcoal, activated

\section{Antiepileptics}

diazepam

phenobarbital powder

injection, $5 \mathrm{mg} / \mathrm{ml}$ in $2 \mathrm{ml}$ ampoule (intravenous or rectal) tablet, $15 \mathrm{mg}$ and $100 \mathrm{mg}$

elixir, $15 \mathrm{mg} / 5 \mathrm{ml}$

\section{Anti-infective dnugs}

\section{Anthelmintics}

niclosamide

pyrentel

chewable tablet, $500 \mathrm{mg}$

chewable tablet, $250 \mathrm{mg}$ (as embonate)

oral suspension $50 \mathrm{mg}$ (as embonate) $/ \mathrm{ml}$

\section{Antibacterial}

amoxicillin

phenoxymethylpenicillin

capsule or tablet, $250 \mathrm{mg}, 500 \mathrm{mg}$ (anhydrous)

tablet. $250 \mathrm{mg}$ (as potassium salt)

powder for oral suspension, $250 \mathrm{mg}$ (as potassium salt) $/ 5 \mathrm{ml}$ 
procaine

benzylpenicillin

benzathine benzyl

penicillin

erythromycin

sulfamethoxazole +

trimethoprime

\section{Antifungal drugs}

griseofulvin

nystatin powder for injection , I million IU

powder for injection , $1.44 \mathrm{~g}$ benzylpenicillin $(=2.4$ million

IU) in vial

capsule or tablet.250 mg (as ethylsuccinate )

tablet, $100 \mathrm{mg}+20 \mathrm{mg}, 400 \mathrm{mg}+80 \mathrm{mg}$

oral suspension. $200 \mathrm{ng}+40 \mathrm{mg} / 5 \mathrm{ml}$

tablet, $125 \mathrm{mg}, 250 \mathrm{mg}$

tablet, $100000 \mathrm{IU}, 5000001 \mathrm{U}$

pessary, 100000 IU

\section{Antiprotozoal drugs}

Antiamoebic and antigiardiasis drugs

metronidazole

tablet,200mg,500mg

oral suspension, $200 \mathrm{mg}$ (as benzozte) $/ 5 \mathrm{ml}$

\section{Antimalarial drugs}

chloroquine

tablet 150nig(as phosphate or sulfate)

syrup, $50 \mathrm{mg}$ (as phosphate or sulfate) $/ 5 \mathrm{ml}$

primaquine

quinine

tablet, $7.5 \mathrm{mg}, 15 \mathrm{mg}$ (as diphosphate)

tablet, $300 \mathrm{mg}$ (as bisulfate or sulfate)

injecition,300mg (as dihydrochloride) $/ \mathrm{ml}$ in $2 \mathrm{nl}$ ampoule

\section{Antimigranine drugs}

for treatinent of acute attack

acetylsalicylic acid

paracetamol

tablet, 500mg

tablet, 500ming

\section{Drugs affecting the blood}

\subsection{Antianaemia drugs}

ferrous salt+folic acid

tablet, $60 \mathrm{mg}+0.25 \mathrm{mg}$

\section{Blood product and plasma substitutes}

\section{Plasnı substitutes}

dextran 70

injectable solution, $6 \%$

\section{Cardiovascular drugs}

\section{Antianginal drugs}

glyceryl trinitrate

Antihypertensive drugs

hydrochlorothiazide

methyl dopa

nifidipine

tablet,(sublingual), $0.5 \mathrm{mg}$

tablet, $25 \mathrm{mg}$

tablet, $250 \mathrm{mg}$

capsule, $10 \mathrm{mg}$ 
Cardiac glycoside

digoxin

11. Dermatological drugs

Antifungal drugs (topical)

benzoic acid+salicylic

acid

\section{Antiinfective drugs}

methylrosanilinium

(gention violet)

neomycin + bacitracin

silver sulfadiazine tablet, $0.25 \mathrm{mg}$

ointment, $6 \%+3 \%$

aqueous solution, $0.5 \%$ chloride tincture, $0.5 \%$

ointment, $5 \mathrm{mg}$ neomycin sulfate $+500 \mathrm{IU}$ bacitracin zinc $/ \mathrm{g}$ cream, $1 \%$ in 500-g container

Anti-inflammatory and antipruritic drugs

calamine lotion

lotion

Scabicides and pediculicides

benzyl benzoate

lotion, $25 \%$

\section{Disinfectants and antiseptics}

\section{Antiseptics}

chlorhexidine

hydrogen peroxide

polyvidon iodine

methylated spirit

\section{Disinfectants}

calcium hypochlorite

\section{Diuretics}

hydrochlorothiazide

\section{Gastrointestinal drugs}

Antacids and other antiulcer drugs

aluminium hydroxide+

magnesium hydroxide solution, $5 \%$ (diglugonate) for dilution

solution, $3 \%$

solution, containing $0.5 \%$ and $1 \%$ iodine

methylated spirit containing 5\% methyl alcohol and $95 \%$ ethyl

alcohol

powder, ( $70 \%$ available chlorine) for solution

tablet, $25 \mathrm{mg}, 50 \mathrm{mg}$

tablet, 500mg

\section{Antiemetic drugs}

metoclopramide

tablet, 10mg (as hydrochloride)

oral solution, $5 \mathrm{mg}$ (ashydrochloride) $5 / \mathrm{ml}$

injection, $5 \mathrm{mg}$ (as hydrochloride) $/ \mathrm{ml}$ in $2 \mathrm{ml}$ ampoule 
intilhaemorrhoidal drugs

local anaesthetic,

ointment or suppository astringent and anti-inflammatory drugs

Autispasmodic drugs

hyoscine

tablet, 10mg (butylbromide)

(compound preparation of hyoscine is not asvised)

Cathartic drugs

senna

tablet, $7.5 \mathrm{mg}$ (sennosides)

Drugs used in diarrhoea

Oral rehydration

oral rehydration salts

powder, $27.9 \mathrm{~g} / 1$

('omponents

$g$ litre

sodium chloride

3.5

trisodium citrate dihydrate $\quad 2.9$

potassium chloride $\quad 1.5$

glucose

20

15. Hormones, other endocrine drugs

Adrenal hormones and synthetic substitutes'

hydrocortisone

powder for injection, $100 \mathrm{mg}$ (as sodium succinate) in vial

\section{6.immunologicals}

Vaccines should be in line with national Extended Programme of Immunisation (EPI).

\section{Ophthalnological prep:trations}

Anti-infective agents

gentamicin

silver nitrate

tetracycline

\section{Oxytocics and antioxytocics}

\section{Oxytocics}

ergometrine

oxytocine solution,(eye drops), $0.3 \%$

solution,(eye drops), $1 \%$

eye ointment $1 \%$, (hydrochloride)

(only in the presence of $\mathrm{MCH}$ clinic)

\section{Antioxytocics}

salbutamol

tablet, $0.2 \mathrm{mg}$ (hydrogen maleate)

injection, $0.2 \mathrm{mg}$ (hydrogen maleate) in $1-\mathrm{ml}$ ampoule

injection, $10 \mathrm{IU}$ in $1 \mathrm{ml}$ ampoule

(only in the presence of MCH clinic)

tablet, $4 \mathrm{mg}$ (as sulfate) 
19. Psychotherapeutics drugs

diazepam

tablet, $2 \mathrm{mg}$, $5 \mathrm{mg}$

20. Drug acting on the respiratory tract

Antiasthmatic drugs

aminophylline

tablet , 100mg, 200nig

injection, $25 \mathrm{mg} / \mathrm{ml}$ in $10 \mathrm{ml}$ ampoule epinephrine

injection, $1 \mathrm{mg}$ (as hydrochloride) in $1 \mathrm{ml}$ ampoule

salbutamol

tablet, $2 \mathrm{mg}$, $4 \mathrm{mg}$ (as sulfate)

syrup, $2 \mathrm{mg}$ (as sulfate) $/ 5 \mathrm{nll}$

\section{Antitussives}

dextromethorphan

syrup, $15 \mathrm{mg}$ (hydrobronide) $/ 5 \mathrm{ml}$

21. Solutions correcting water, electrolyte and acid-base disturbances

Oral rehydration

oral rehydration salts

('omponents

sodium chloride

trisodium citrate dihydrate

potassium chloride

glucose

\section{Parenteral}

sodium chloride

compound solution of sodium lactate

\section{Miscellaneous}

water for injection

\section{Vitamins and minerals}

ascorbic acid

calcium

ergoclciferol

iodine

retinol powder, $27.9 \mathrm{~g} / \mathrm{l}$

$g$ litre

3.5

2.9

1.5

20

injectable solution, $0.9 \%$ isotonic (equivalent to $\mathrm{Na} 154 \mathrm{mn} 1 \mathrm{l} / \mathrm{l}$,

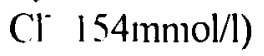

injectable solution

$2 \mathrm{ml}, 5 \mathrm{ml}$ ampoule

tablet , $50 \mathrm{mg}$

tablet, 500mig(lactate)

capsule or tablet, $1.25 \mathrm{mg}$ (50 000 IU) oral solution, $0.25 \mathrm{mg}$ $/ \mathrm{ml}(10000 \mathrm{lU} / \mathrm{ml})$

iodized oil, I ml (480my iodine),

capsule, $200 \mathrm{mg}$

sugar coated tablet, 10000 IU ( as palmitate) $(5.5 \mathrm{mg})$

capsule, $200000 \mathrm{IU}$ ( as palmitate)( $110 \mathrm{mg}$ ) 
DRUGS USED AT SUB CENTRE/C2

(prescribers: medical assistants \& med-level health workers with more than one year medical education)

1. Analgesics, antipyretics, non-steroid anti inflammatory drugs

acetylsalicylic acid

tablet, 300, 500mg

paracetamol

tablet, $100,500 \mathrm{mg}$

syrup, $125 \mathrm{mg} / 5 \mathrm{ml}$

2.Antiallergics and drugs used in anaphylaxis

chlorphenamine

tablet 4mg (hydrogen maleate)

3. Antidote and other substances used in poisonings

charcoal, activated

powder

\section{Anti-infective drugs}

\section{Anthelmintics}

pyrentel

chewable tablet,250mg(as embonate)

oral suspension $50 \mathrm{mg}$ (as embonate) $/ \mathrm{ml}$

\section{Antibacterial}

amoxicillin

phenoxymethyl

capsule or tablet, $250 \mathrm{mg}$, (anhydrous)

penicillin

erythromycin

sulfamethoxazole +

trimethoprime

tablet, $250 \mathrm{mg}$ (as potassium salt)

powder for oral suspension, $250 \mathrm{mg}$ (as potassium salt) $/ 5 \mathrm{ml}$

capsule or tablet, $250 \mathrm{mg}$ (as ethylsuccinate )

tablet, $100 \mathrm{mg}+20 \mathrm{mg}, 400 \mathrm{mg}+80 \mathrm{mg}$ oral suspension, $200 \mathrm{mg}$

$+40 \mathrm{nig} / 5 \mathrm{ml}$

Antifungal drugs

nystatin

tablet, 100000 IU

Antiprotozoal drugs

Antiamoebic and antigiardiasis drugs

metronidazole

tablet, $200 \mathrm{mg}, 500 \mathrm{mg}$

oral suspension, $200 \mathrm{mg}$ (as benzozte) $/ 5 \mathrm{ml}$

Antimalarial drugs

chloroquine

tablet $150 \mathrm{mg}$ (as phosphate or sulfate)

syrup, $50 \mathrm{mg}$ (as phosphate or sulfate) $/ 5 \mathrm{ml}$

5. Drugs affecting the blood

ferrous salt + folic acid

tablet, $60 \mathrm{mg}+0.25 \mathrm{mg}$ 


\section{Dermatological drugs}

Antiinfective drugs

methylrosanilinium

aqueous solution, $0.5 \%$

chloride

tincture, $0.5 \%$

(gention violet)

silver sulfadiazine

cream, $1 \%$ in 500 -g container

Anti-inflammatory and antipruritic drugs

calamine lotion

Iotion

Scabicides and pediculicides

benzyl benzoate

Iotion , $25 \%$

\section{Disinfectants and antiseptics}

\section{Antiseptics}

chlorhexidine

solution, $5 \%$ (diglugonate) for dilution

polyvidon iodine

solution, containing $0.5 \%$ and $1 \%$ iodine

\section{Disinfectants}

calcium hypochlorite

powder, (70\% available chlorine) for solution

\section{Gastrointestinal drugs}

Antacids and other antiulcer drugs

aluminium hydroxide+

tablet, 500mg

magnesium hydroxide

\section{Antiemetic drugs}

metoclopramide

tablet, 10mg (as hydrochloride)

\section{Antihaemorrhoidal drugs}

local anaesthetic,

ointment or suppository

astringent and

anti-inflammatory drugs

Cathartic drugs

senna

tablet, 7.5mg(sennosides)

\section{Drugs used in diarrhoea}

Oral rehydration

oral rehydration salts

powder, $27.9 \mathrm{~g} / 1$

('omponents.

g lire

sodium chloride

trisodium citrate dihydrate 
Anti-infective agents

silver nitrate

tetracycline

10. Oxytocics

ergometrine solution,(eye drops), $1 \%$

eye ointment $1 \%$, (hydrochloride)

11. Drug acting on the respiratory tract

\section{Antiasthmatic drugs}

aminopliylline

\section{Antitussives}

dextromethorphan tablet , 100mg,200nig

injection, $25 \mathrm{mg} / \mathrm{ml}$ in $10 \mathrm{ml}$ ampoule

syrup, $15 \mathrm{mg}$ (hydrobronuide)/5ml

12. Solutions correcting water, electrolyte and acid-base disturbances

oral rehydration salts

(omponents

sodium chloride

trisodium citrate dihydrate

potassium chloride

glucose

\section{Vitamins and minerals}

ascorbic acid

ergociciferol

calcium

iodine powder, $27.9 \mathrm{~g} / \mathrm{l}$

$g$ litre

3.5

2.9

1.5

20

tablet ,50 mg

capsule or tablet, $1.25 \mathrm{mg}$ (50 $000 \mathrm{IU}$ ) oral solution, $0.25 \mathrm{mg}$ $/ \mathrm{ml}(100001 \mathrm{U} / \mathrm{ml})$

tablet, $500 \mathrm{mg}$ ( lactate )

iodized oil, $1 \mathrm{ml}$ (480mg iodine),

capsule, $200 \mathrm{mg}$ 